\title{
Multiple Eyelid Signs are Suggestive of Demodex Infestation
}

\author{
This article was published in the following Dove Press journal: \\ Clinical Ophthalmology
}

\section{Lunla Udomwech (iD) Nonthapan Phasuk (D)}

School of Medicine, Walailak University, Nakhon Si Thammarat, Thailand
Correspondence: Lunla Udomwech School of Medicine, Walailak University, 222 Thaiburi, Tha Sala, Nakhon Si Thammarat, 80160, Thailand

Tel +667567280 I

Fax +6675672807

Email lunla.ud@wu.ac.th
Purpose: To determine the signs associated with Demodex infestation in blepharitis patients and the rates of eyelash Demodex colonization among blepharitic patients and healthy individuals in Thailand.

Patients and Methods: This was a prospective, case-control clinical study. Eighty-three controls and 43 blepharitic patients were consecutively enrolled from Walailak University Hospital. Each patient answered a questionnaire, underwent a complete eye exam, and had four lashes from each eye epilated; eyelashes were directly examined under a light microscope. Ocular signs, including types of eyelash debris (waxy debris, scaly debris, or cylindrical dandruff (CD)), erythema and telangiectasia of the eyelid, were noted.

Results: Demodex infestation was observed more frequently among patients with blepharitis than among controls $(67.4 \%$ in the blepharitis group, $26.5 \%$ in the control group, p-value $<0.001)$. The participants with Demodex infestation were 5.7 times more likely to have blepharitis than the controls (odds ratio 5.74; 95\% confidence interval 2.57-12.82, p-value $<0.001$ ). Clinical signs that were significantly ( $p$-value $<0.05$ ) related to demodicosis were lid erythema, lid telangiectasia, and any type of eyelash debris (scaly, waxy, or CD).

Conclusion: In contrast to previous studies that found only CD to be highly associated with eyelash demodicosis, we found multiple suggestive signs: any kind of debris on eyelashes (scaly, waxy, or CD), eyelid erythema, and eyelid telangiectasia. Approximately one-quarter $(26.5 \%)$ of the studied population had asymptomatic mite colonization. Demodex infestation should therefore always be on the list of possible etiologies when treating patients with blepharitis or other ocular surface-related problems.

Keywords: Demodex, blepharitis, eyelid signs, debris, Thailand

\section{Introduction}

Blepharitis is a condition of eyelid inflammation and can be subcategorized as anterior and posterior blepharitis. Anterior blepharitis refers to inflammation of the area involving the eyelashes and skin on the anterior part of the eyelid margin, while posterior blepharitis refers to meibomian gland pathology. Blepharitis causes ocular discomfort, such as dry eye, photophobia, epiphora, blurred vision, and foreign body sensation; all are common chief complaints in ophthalmology clinics. Severity can vary from asymptomatic to severe cases with secondary keratitis. Blepharitis has multiple etiologies, including systemic conditions such as rosacea and seborrheic dermatitis, bacterial infection, or parasitic infestation, especially by Demodex spp. ${ }^{1,2}$

Demodex spp. belongs to the family Demodicidae of the class Arachnida in the phylum Arthropoda. These mites are common obligate, host-specific, human 
ectoparasites that reside in hair follicles. ${ }^{3}$ Two species that infest humans have been identified. The longer and narrower mite is Demodex folliculorum, which is typically found in the infundibulum of a hair follicle, and the shorter mite is Demodex brevis, which is typically found deep in the hair follicle. ${ }^{4,5}$

Demodex infestation can cause both anterior and posterior blepharitis, dry eye, rosacea, and hordeolum and can even mimic sebaceous gland carcinoma. ${ }^{6,7}$ Its varying manifestations and multifactorial nature may mislead ophthalmologists and delay diagnosis and appropriate treatment. In patients with anterior blepharitis, the eyelid margin and the eyelashes often appear to harbor debris. One specific type of debris that appears as a collar wrapping around the base of an eyelash, namely, cylindrical dandruff (CD) or collarettes, has been found to be a highly specific indicator of ocular demodicosis. ${ }^{8,9}$

The prevalence of ocular demodicosis varies greatly among different studies, ranging from $4 \%$ to $47 \%{ }^{8,10-13}$ All ethnicity and age groups can be affected, with increased susceptibility in those with advanced age. ${ }^{9}$ Since the discovery of these mites, many studies have shown a correlation between infestation and ocular pathology. Signs and symptoms can be alleviated with treatment. However, no direct cause-effect relationship has yet been successfully demonstrated. Since Demodex spp. cannot survive long separated from their human hosts and in vitro culture has not been achieved, their life cycle and pathophysiology are not fully understood. ${ }^{3,14}$ Its presence on individuals with healthy eyes also has raised questions regarding the possibility of a nonpathogenic role in the microenvironment around the ocular surface. ${ }^{15,16}$ Some researchers have proposed that mites may be normal ocular surface fauna (commensalism with humans) that can turn pathogenic under overpopulation conditions related to an altered microenvironment. ${ }^{3-5,8,16,17}$

In Thailand, only one investigation focusing on the prevalence of Demodex has been performed. The study, which was performed in a tertiary care hospital, reported a prevalence of $42 \% .{ }^{12}$ Having data from two tertiary hospitals and smaller hospitals (in this study) will help health professionals understand the magnitude of this condition. This study aimed to determine the signs associated with Demodex infestation in blepharitis patients and determine the rates of eyelash Demodex colonization among blepharitic patients and healthy individuals in Thailand.

\section{Materials and Methods}

\section{Ethics Approval}

All the tenets of the Declaration of Helsinki were adhered to. The study was reviewed and approved by the Ethics Committee on Human Rights Related to Research Involving Human Subjects, Walailak University, prior to the recruitment of participants (WUEC-16-139-01).

\section{Consent to Participate}

All participants provided informed consent, and when appropriate: assent, prior to enrollment.

\section{Study Design and Setting}

This prospective, case-control study was carried out from April 2018 to April 2019 in the Ophthalmology Clinic, Walailak University Hospital. Walailak University Hospital is located in Tha Sala district, Nakhon Si Thammarat Province, southern Thailand, approximately $780 \mathrm{~km}$ from the Thai capital of Bangkok. The hospital was established in 2017 as the pilot for an official 800-bed university hospital.

\section{Sample Size and Study Population}

The study population consisted of patients aged between 18 and 89 years who attended the ophthalmology clinic between April 2018 and April 2019. Investigators planned a study of independent cases and controls, with two controls per case. Prior data indicated that the probability of exposure among cases and controls was 0.2972 and 0.0909, respectively. ${ }^{18}$ By using a case-control study design, 40 case patients and 80 control patients were required to reject the null hypothesis that the exposure rates for case and controls have a probability of being equal (power of 0.8 ). The type I error probability associated with this null hypothesis test was 0.05 . We used an uncorrected chi-squared statistic to evaluate this null hypothesis. Finally, a total of 43 patients and 83 controls were willing to participate in the study, and we decided to enroll all the samples in the study for the purpose of health surveillance regardless of the calculated sample size. The inclusion criteria for case were age of 18-89 years and being diagnosed with blepharitis at the ophthalmology clinic. The controls in this study were individuals interested in an advertisement at the outpatient department or approached by a research assistant at ophthalmology clinic, Walailak University Hospital. Cases and controls were matched based on age and sex. After the desired number of cases was collected, the total number of cases in 
each age group, separating male and female cases, was matched for twice the number of controls, resulting in 2 controls per 1 case with age range and sex matched. Individuals who or topical ocular antibiotics within 14 days prior to examination or used systemic antibiotics within 1 month or were previously diagnosed or treated for demodicosis were excluded from the study.

\section{Questionnaire and Eye Examination}

Each patient answered the Standardized Patient Evaluation of Eye Dryness (SPEED) questionnaire and received a comprehensive eye exam, including best-corrected visual acuity (BCVA), which was assessed with an early treatment for diabetic retinopathy study (ETDRS)-type visual acuity (VA) chart and measured at $4 \mathrm{~m}$, intraocular pressure (IOP) exam (Nidek NT-530P, Japan), and an anterior segment exam (Haag-Streit BQ900, Switzerland) including fluorescein staining of the tear film to measure the staining pattern of the cornea and conjunctiva and tear break-up time.

The presence of debris attached to eyelashes was classified based on its physical appearance as cylindrical (Figure 1), scaly (Figure 2), or waxy (Figure 3). Cylindrical dandruff was defined as accumulation of materials, wrapping around the base of eyelashes in the shape
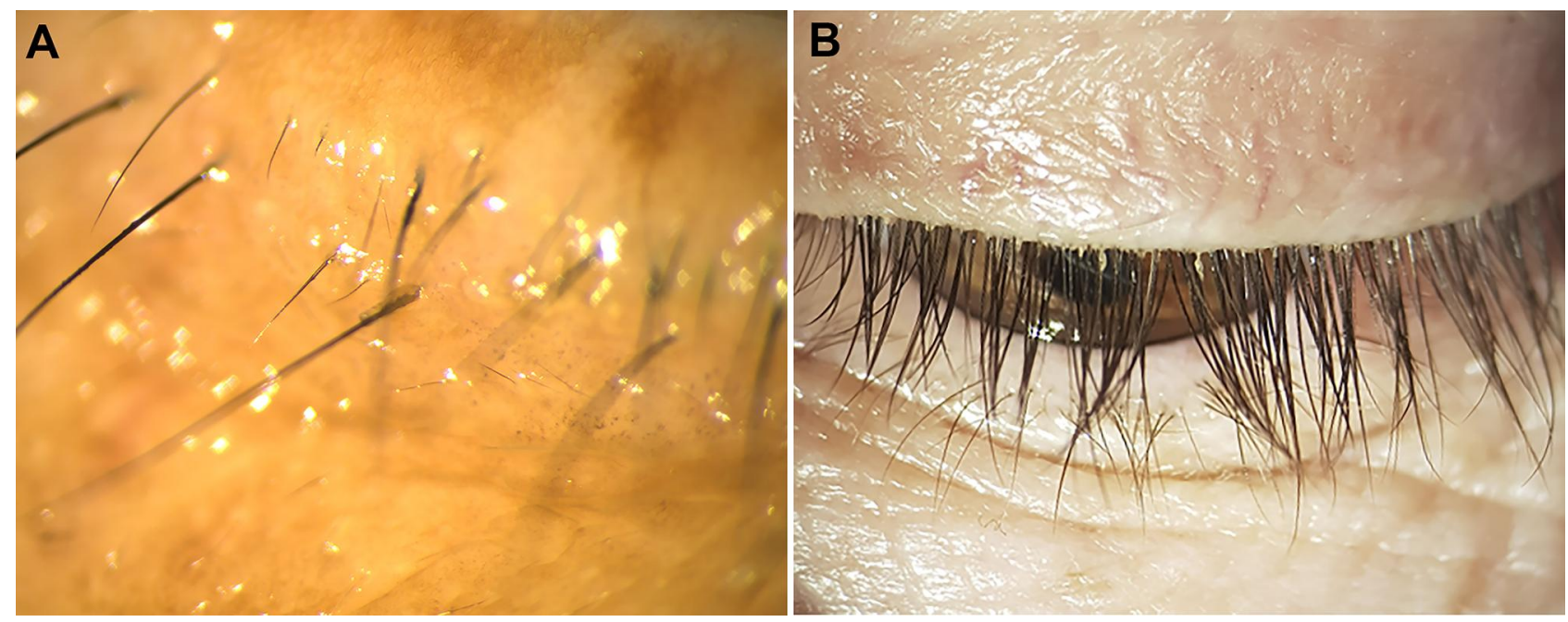

Figure I Slit-lamp photograph of cylindrical dandruff attached around the base of eyelashes. A: Magnified view of single cylindrical dandruff at the center of the figure. B: An overall view of a patient with multiple cylindrical dandruff adhered to the base of eyelashes.

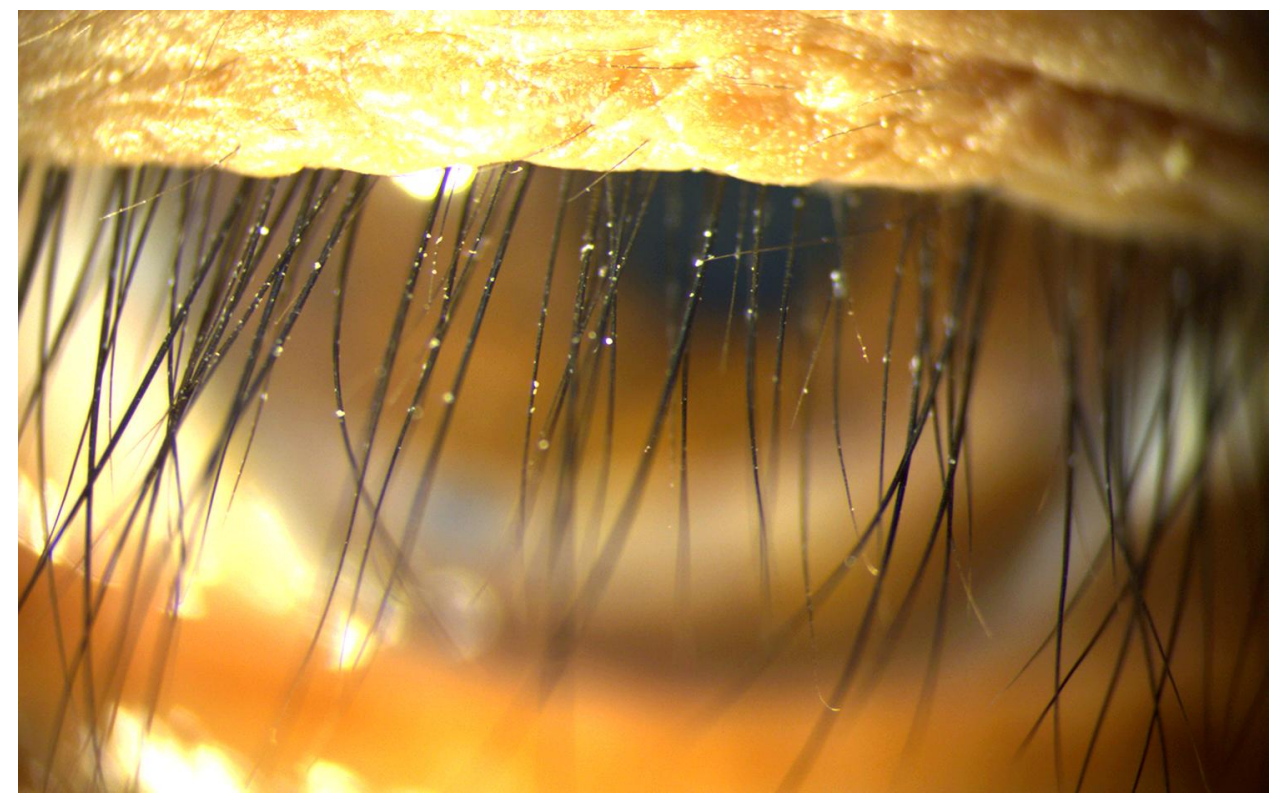

Figure 2 Slit-lamp photograph of scaly debris, appeared as flakes adhered on eyelashes. 

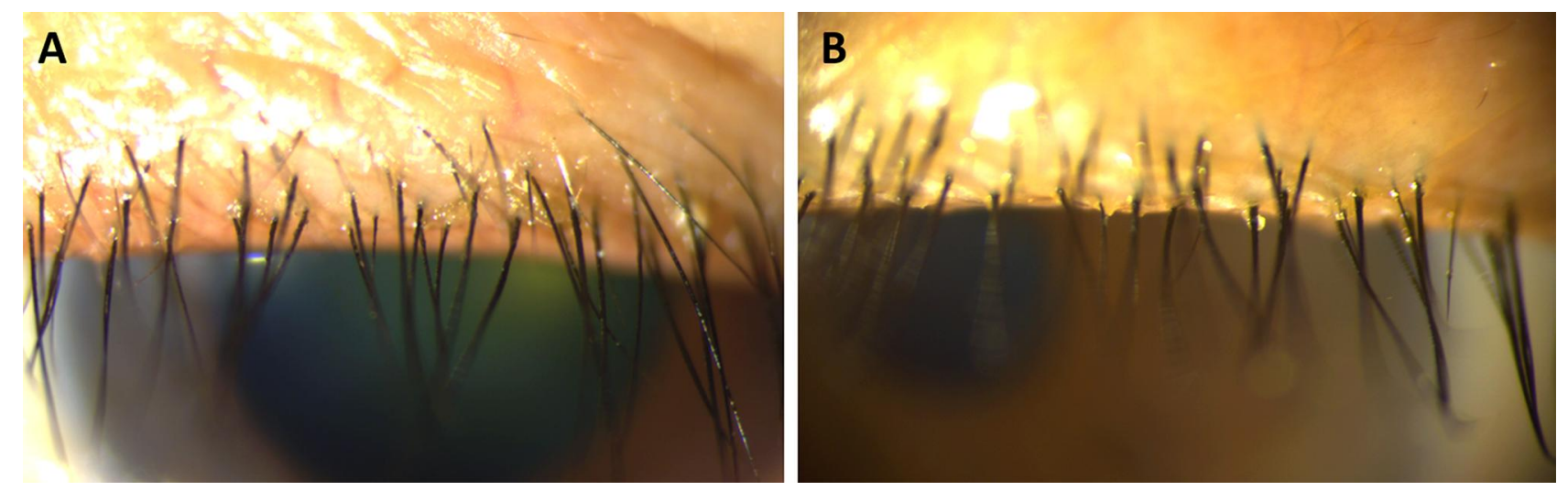

Figure 3 Slit-lamp photograph of waxy debris, appeared as lipid on eyelid margin and base of eyelashes.

of cylinders or collars. ${ }^{8}$ Scaly debris was flaky materials adhered to eyelashes or eyelid margin. Waxy debris was characterized by greasy material attaching to eyelashes or eyelid margin without taking the shape of cylinders. Eyelid erythema, telangiectasia, and the presence of meibomian gland dysfunction were also noted. All clinical examinations were performed by the primary investigator (LU) to ensure consistency of all clinical findings.

\section{Detection of Demodex spP}

Four eyelashes from each eye were epilated using cilia forceps. When eyelash debris was present, the eyelashes with the most debris were the priority candidates for epilation. When no eyelash debris was present, four random lashes from each eye were sampled. The lashes were placed on glass slides in one drop of $0.2 \% \mathrm{w} / \mathrm{w}$ carbomer gel (Vidisic ${ }^{\circledR}$ Gel, Bausch \& Lomb GmbH, Berlin, Germany) and covered with a coverslip. The specimens were then examined under a light microscope by a certified medical technician. The presence and number of mites observed were reported (Figure 4).

\section{Statistical Analysis}

Data were entered, cleaned, and analyzed using IBM SPSS Statistics for Windows, Version 23. Data in this study were normally distributed as determined by the KolmogorovSmirnov test. Quantitative variables were described by means and standard deviations (SDs), and qualitative variables were described by frequencies (percentages). A chisquare test was used to compare qualitative variables (i.e., age groups, clinical findings on lids), and an independent $t$-test was run to compare the means of qualitative variables. The risk of blepharitis in the participants with Demodex infestation was determined by univariable

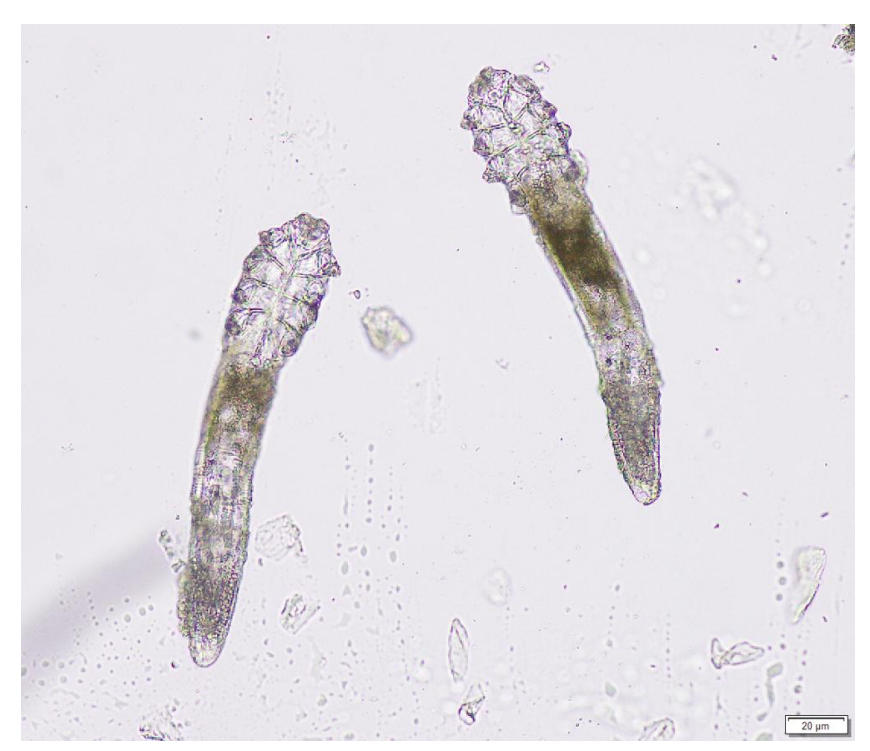

Figure 4 Demodex spp. from light microscopy.

logistic regression analysis. Differences were considered to be statistically significant when the p-value was less than 0.05 .

\section{Results}

A total of 126 individuals were included in the study: 83 healthy participants were enrolled in the control group, and 43 patients with blepharitis were enrolled in the blepharitis group. No statistically significant difference in demographic data was found between the blepharitis and control groups (age; sex; predisposing nonocular medical conditions, such as diabetes mellitus, hypertension, and dyslipidemia; VA; and IOP). However, patients in the blepharitis group reported significantly higher rates of previously diagnosed dry eye syndrome and use of ocular medication than patients in the control group (Table 1). 
Table I Demographic Data of the Participants in Blepharitis and Control Groups

\begin{tabular}{|c|c|c|c|}
\hline & Blepharitis $(n=43)$ & Controls $(n=83)$ & p-value \\
\hline Age (mean, SD) & $52.44,16.89$ & $52.65,16.86$ & $0.948^{\mathrm{b}}$ \\
\hline \multicolumn{4}{|l|}{ Age group } \\
\hline $10-19, n(\%)$ & $0(0 \%)$ & $2(2.4 \%)$ & \multirow[t]{8}{*}{$0.989^{\mathrm{a}}$} \\
\hline 20-29, n(\%) & $3(7.0 \%)$ & 7 (8.4\%) & \\
\hline $30-39, n(\%)$ & $4(9.3 \%)$ & 7 (8.4\%) & \\
\hline $40-49, n(\%)$ & $12(27.9 \%)$ & $21(25.3 \%)$ & \\
\hline $50-59, n(\%)$ & 7 (16.3\%) & $14(16.9 \%)$ & \\
\hline $60-69, n(\%)$ & $9(20.9 \%)$ & $16(19.3 \%)$ & \\
\hline 70-79, n(\%) & 7 (16.3\%) & 14 (I6.9\%) & \\
\hline $80-89, n(\%)$ & I $(2.3 \%)$ & $2(2.4 \%)$ & \\
\hline Female gender & $33(76.7 \%)$ & $67(80.7 \%)$ & $0.60 I^{a}$ \\
\hline Diabetes mellitus, $\mathrm{n}(\%)$ & $4(9.3 \%)$ & $7(8.4 \%)$ & $0.870^{\mathrm{a}}$ \\
\hline Hypertension, n(\%) & $7(16.3 \%)$ & $15(18.1 \%)$ & $0.802^{\mathrm{a}}$ \\
\hline Dyslipidemia, n(\%) & 7 (16.3\%) & $8(9.6 \%)$ & $0.275^{\mathrm{a}}$ \\
\hline Dry eyes, n(\%) & $21(48.8 \%)$ & $20(24.1 \%)$ & $0.005^{\mathrm{a} *}$ \\
\hline Glaucoma, n(\%) & $2(4.7 \%)$ & $3(3.6 \%)$ & $0.777^{\mathrm{a}}$ \\
\hline Ocular medications, n(\%) & II (25.6\%) & $6(7.2 \%)$ & $0.004^{\mathrm{a} *}$ \\
\hline Right-LogMAR (mean, SD) & $0.0405,0.070$ & $0.054,0.121$ & $0.499^{b}$ \\
\hline Left-LogMAR (mean, SD) & $0.033,0.079$ & $0.047,0.098$ & $0.434^{\mathrm{b}}$ \\
\hline Right-IOP (mean, SD) & $13.857,3.314$ & 13.340, 3.567 & $0.439^{b}$ \\
\hline Left-IOP (mean, SD) & $13.640,2.780$ & $13.169,2.850$ & $0.383^{b}$ \\
\hline
\end{tabular}

Notes: $*$ Statistical significance $p<0.05$. ${ }^{a}$ Chi-square test. ${ }^{b}$-test.

In total, $67.4 \%$ (29 out of 43 ) of the patients in the blepharitis group were found to be infested with Demodex. (Figure 4) Only 26.5\% (22 out of 83 ) of the controls were found to have Demodex infestation. The association between blepharitis and Demodex infestation was statistically significant (p-value $<0.001$ ). The participants with Demodex infestation were 5.7 times more likely to have blepharitis than the controls (odds ratio 5.74; $95 \%$ confidence interval 2.57-12.82, p-value $<0.001$ ). This also indicates that the prevalence of eyelash demodicosis among healthy Thai individuals is $26.5 \%$ (Table 2).
Comparisons between individuals with Demodex infestation (55 of 126) and those without (75 of 126) revealed no significant differences in age, VA, IOP, or SPEED score. However, clinical findings of lid erythema, lid telangiectasia, and eyelash debris of any kind (cylindrical, scaly, or waxy) were found to be significantly associated with the presence of eyelash Demodex infestation (Table 3).

The blepharitis group not only had more patients with Demodex infestation but also had a higher mite load than the control group. On the basis of the 8-lash samples

Table 2 Association of Demodex Infestation and Blepharitis

\begin{tabular}{|l|c|c|c|c|c|}
\hline & Blepharitis, $\mathbf{n}(\%)$ & Controls, $\mathbf{n}(\%)$ & Odds Ratio & $\mathbf{9 5 \%} \mathbf{C l}^{\mathbf{a}}$ & $\mathbf{p}^{\mathbf{a}} \mathbf{v a l u e}$ \\
\hline Presence of Demodex spp. & $29(67.4)$ & $22(26.5)$ & 5.74 & $2.57-12.82$ & $<0.001$ \\
No Demodex spp. & $14(32.6)$ & $61(73.5)$ & & & \\
Total & 43 & 83 & & \\
\hline
\end{tabular}

Notes: ${ }^{2}$ Confidence interval. ${ }^{b}$ Univariable logistic regression analysis. 
Table 3 Association of Clinical Findings and Demodex Infestation

\begin{tabular}{|l|c|c|c|}
\hline & Presence of Demodex spp. (n=50) & No Demodex spp. (n=75) & p-value \\
\hline Age (mean, SD) & $55,17.143$ & $50.93,16.477$ & $0.183^{\mathrm{b}}$ \\
Right-LogMAR (mean, SD) & $0.056,0.114$ & $0.045,0.102$ & $0.586^{\mathrm{b}}$ \\
Left-LogMAR (mean, SD) & $0.058,0.107$ & $0.032,0.079$ & $0.121^{\mathrm{b}}$ \\
Right-IOP (mean, SD) & $13.790,4.564$ & $13.342,2.542$ & $0.491^{\mathrm{b}}$ \\
Left-IOP (mean, SD) & $13.423,13.272$ & $13.272,2.817$ & $0.774^{\mathrm{b}}$ \\
Lid erythema, n(\%) & $14(28.00 \%)$ & $6(8.00 \%)$ & $0.003^{\mathrm{a} *}$ \\
Lid telangiectasia, n(\%) & $17(34.00 \%)$ & $5(6.67 \%)$ & $<0.001^{\mathrm{a} *}$ \\
Scaly debris, n(\%) & $14(28.00 \%)$ & $10(13.33 \%)$ & $\left.0.04\right|^{\mathrm{a} *}$ \\
Waxy debris, n(\%) & $18(36.00 \%)$ & $11(14.67 \%)$ & $0.006^{\mathrm{a} *}$ \\
Cylindrical dandruff, n(\%) & $13(26.00 \%)$ & $7(9.33 \%)$ & $0.013^{\mathrm{a} *}$ \\
Dry eye score SPEED (mean, SD) & $7.612,5.664$ & $7.867,5.414$ & $0.802^{\mathrm{a}}$ \\
\hline
\end{tabular}

Notes: ${ }^{*}$ Statistical significance $p<0.05$. ${ }^{a}$ Chi-square test. ${ }^{b}$-test.

obtained from each patient, the blepharitis group had a mean load of 3.44 mites per patient, while the control group had a mean load of only 0.72 mites per person. The greatest difference was found in the 30-39 years age group; the blepharitis group showed a maximum infestation of 6.75 Demodex mites per eye, while the agematched controls showed no infestation at all.

\section{Discussion}

In agreement with previous studies, data gathered from this study suggested that eyelash demodicosis was significantly associated with blepharitis. The presence of Demodex mites was significantly associated with blepharitis. The prevalence of eyelash demodicosis in blepharitis patients in this study was $67.4 \%$ (29 of 43), while colonization among otherwise healthy Thai individuals was $26.5 \%$, with a p-value of $<0.001$. One prior study in Thailand directly reported the prevalence of eyelash demodicosis as $42 \%$ according to microscopic examination techniques and 79\% according to seminested PCR. ${ }^{12}$ Another study in Thailand that detected Demodex by epilation and direct examination under a light microscope as part of a dry eye examination reported a $47.6 \%$ infestation rate among controls and a $57.1 \%$ infestation rate in patients who used eyeliner. ${ }^{19}$ Our high rate of Demodex infestation among blepharitic patients, at $67.4 \%$, compared with the previous study's infestation rate of $42-57.1 \%$ using the direct examination method, reflects varying prevalence rates among different populations. Surprisingly, our study found the prevalence of demodicosis among blepharitic patients to be even higher than that detected in a tertiary hospital setting in Bangkok, Thailand. ${ }^{12,19}$ Environmental factors associated with an increased prevalence of ocular demodicosis, such as humidity and temperature, which are high in the current study's region, might affect the presence of Demodex spp.; this needs to be further investigated in the future. Demodex colonization among otherwise healthy individuals has been widely reported. Our $26.5 \%$ colonization rate correlated with those from some other studies, such as the study by Gao et al, who observed Demodex infestation in $22 \%$ of patients with clean eyelashes. However, the prevalence of Demodex infestation varies greatly among studies and populations, ranging from $4 \%$ to $47 \%{ }^{8}$ The presence of Demodex spp. in this study was not related to the age of the participants. In contrast, previous studies conducted in Korea and Poland indicated that the prevalence of Demodex infestation increased with age. ${ }^{20-22}$ There was no relationship between the presence of Demodex spp. and sex in this study, which was in agreement with most previous studies. ${ }^{13,20-22}$ With regards to a mite load, the significance of the 30-39 years age group having the greatest difference of Demodex load between cases and controls is questionable due to the uneven distribution of the number of cases and controls among different age groups.

$\mathrm{CD}$ is a classical hallmark of eyelash Demodex infestation, as reported by Gao et al, who found Demodex infestation in all cases with $\mathrm{CD}^{8}$ A previous study in a Thai population by Kasetsuwan et al found that $69 \%$ of individuals with Demodex infestation exhibited CD, while only $15.5 \%$ of those without Demodex infestation exhibited $\mathrm{CD}$ on eyelashes. ${ }^{12}$ In our study, a much lower proportion, $26 \%$, of patients with demodicosis showed CD on examination, while $9.33 \%$ of individuals without Demodex infestation also had CD. Data from our study revealed that 
various clinical signs are suggestive of the disease, which is a different perspective on the clinical manifestations of Demodex spp.-associated blepharitis.

Our results showed that not only CDs but also various eyelid signs, including lid erythema (Demodex 28\%, no Demodex 8\%, $\mathrm{p}=0.003$ ), lid telangiectasia (Demodex $34 \%$, no Demodex $6.67 \%$, p $<0.001)$, scaly debris (Demodex 28\%, no Demodex 13.33\%, p $=0.041$ ), and waxy debris (Demodex 36\%, no Demodex 14.67\%, p = $0.006)$ were significantly associated with eyelash demodicosis (Table 3).

One counterintuitive finding was the SPEED scores from questionnaires. The Demodex infestation group had a score of $7.612( \pm 5.564)$, while the control group had a mean score of $7.867( \pm 5.414)(\mathrm{p}=0.802)$. This finding might result from the complex multifactorial nature of dry eye syndrome. Although many ocular signs were statistically significant indicators of Demodex infestation, the symptoms evaluated by the SPEED questionnaire did not show any associations with mite infestation.

\section{Conclusion}

Demodex infestation was significantly associated with blepharitis, and several signs are valuable clinical clues. Signs that are suggestive of Demodex infestation are any kind of debris on the eyelashes, including cylindrical dandruff, scaly debris, or waxy debris; eyelid erythema; and eyelid telangiectasia. Eyelash Demodex infestation can occur in any age group. More than a quarter $(26.5 \%)$ of the population can have asymptomatic mites colonization. Demodex infestation is common and therefore should always be on the list of possible etiologies when treating patients with blepharitis or other ocular surface-related problems. Treatment of demodicosis in patients with clear signs and symptoms is advised; however, in patients with asymptomatic colonization, no consensus has been established. Further research should elucidate the mites' life cycles, behavior, biochemical and immunological interactions, and their effect on the microenvironment of the human eye in various situations.

\section{Abbreviations}

CD, cylindrical dandruff; SPEED, Standardized Patient Evaluation of Eye Dryness; BCVA, best-corrected visual acuity; ETDRS, early treatment for diabetic retinopathy study; VA, visual acuity; IOP, intraocular pressure exam; SDs, standard deviations.

\section{Disclosure}

This work was supported by Walailak University (grant number WU-IRG61-03) as means for the resources of this research, but not for the authors' personal expenses. The funder had no role in study design, data collection and analysis, decision to publish, or preparation of the manuscript. The authors report no conflicts of interest in this work.

\section{References}

1. Sarac G, Cankaya C, Ozcan KN, Cenk H, Kapicioglu YK. Increased frequency of Demodex blepharitis in rosacea and facial demodicosis patients. J Cosmet Dermatol. 2020;19(5):1260-1265. doi:10.1111/ jocd. 13150

2. Fromstein SR, Harthan JS, Patel J, Opitz DL. Demodex blepharitis: clinical perspectives. Clin Optom. 2018;10:57-63. doi:10.2147/ OPTO.S142708

3. Liu J, Sheha H, Tseng SC. Pathogenic role of Demodex mites in blepharitis. Curr Opin Allergy Clin Immunol. 2010;10(5):505-510. doi:10.1097/ACI.0b013e32833df9f4

4. Bhandari V, Reddy JK. Blepharitis: always remember demodex. Middle East Afr J Ophthalmol. 2014;21(4):317-320. doi:10.4103/ 0974-9233.142268

5. Kheirkhah A, Blanco G, Casas V, Tseng SC. Fluorescein dye improves microscopic evaluation and counting of demodex in blepharitis with cylindrical dandruff. Cornea. 2007;26(6):697-700. doi:10.1097/ICO.0b013e31805b7eaf

6. Galea M, Sharma R, Srinivasan S, Roberts F. Demodex blepharitis mimicking eyelid sebaceous gland carcinoma. Clin Exp Ophthalmol. 2014;42(2):208-210. doi:10.1111/ceo.12116

7. Luo X, Li J, Chen C, Tseng S, Liang L. Ocular demodicosis as a potential cause of ocular surface inflammation. Cornea. 2017;36 (Suppl 1):S9. doi:10.1097/ICO.0000000000001361

8. Gao YY, Di Pascuale MA, Li W, et al. High prevalence of Demodex in eyelashes with cylindrical dandruff. Invest Ophthalmol Vis Sci. 2005;46(9):3089-3094. doi:10.1167/iovs.05-0275

9. Lopez-Ponce D, Zuazo F, Cartes C, et al. High prevalence of Demodex spp. infestation among patients with posterior blepharitis: correlation with age and cylindrical dandruff. Arch Soc Esp Oftalmol. 2017;92(9):412-418. doi:10.1016/j.oftal.2017.01.001

10. Zhao YE, Wu LP, Hu L, Xu JR. Association of blepharitis with Demodex: a meta-analysis. Ophthalmic Epidemiol. 2012;19 (2):95-102. doi:10.3109/09286586.2011.642052

11. Czepita D, Kuzna-Grygiel W, Czepita M, Grobelny A. Demodex folliculorum and Demodex brevis as a cause of chronic marginal blepharitis. Ann Acad Med Stetin. 2007;53(1):63-67; discussion 67.

12. Kasetsuwan N, Kositphipat K, Busayarat M, et al. Prevalence of ocular demodicosis among patients at tertiary care center, Bangkok, Thailand. Int $J$ Ophthalmol. 2017;10(1):122-127. doi:10.18240/ ijo.2017.01.20

13. Kemal M, Sumer Z, Toker MI, Erdogan H, Topalkara A, Akbulut M. The prevalence of Demodex folliculorum in blepharitis patients and the normal population. Ophthalmic Epidemiol. 2005;12(4):287-290. doi:10.1080/092865805910057

14. Rufli T, Mumcuoglu Y. The hair follicle mites Demodex folliculorum and Demodex brevis: biology and medical importance. A review. Dermatologica. 1981;162(1):1-11. doi:10.1159/00025 0228

15. Nicholls SG, Oakley CL, Tan A, Vote BJ. Demodex species in human ocular disease: new clinicopathological aspects. Int Ophthalmol. 2017;37(1):303-312. doi:10.1007/s10792-016-0249-9 
16. Post CF, Juhlin E. Demodex folliculorum and blepharitis. Arch Dermatol. 1963;88:298-302. doi:10.1001/archderm.1963.015902100 56008

17. Cheng AM, Sheha H, Tseng SC. Recent advances on ocular Demodex infestation. Curr Opin Ophthalmol. 2015;26(4):295-300. doi:10.1097/ICU.0000000000000168

18. Turk M, Ozturk I, Sener AG, Kucukbay S, Afsar I, Maden A. Comparison of incidence of Demodex folliculorum on the eyelash follicule in normal people and blepharitis patients. Turkiye Parazitol Derg. 2007;31(4):296-297.

19. Prabhasawat P, Chirapapaisan C, Chitkornkijsin C, Pinitpuwadol W, Saiman M, Veeraburinon A. Eyeliner induces tear film instability and meibomian gland dysfunction. Cornea. 2020;39(4):473-478. doi:10.1097/ICO.0000000000002198
20. Lee SH, Chun YS, Kim JH, Kim ES, Kim JC. The relationship between demodex and ocular discomfort. Invest Ophthalmol Vis Sci. 2010;51(6):2906-2911. doi:10.1167/iovs.09-4850

21. Sedzikowska A, Oseka M, Skopinski P. The impact of age, sex, blepharitis, rosacea and rheumatoid arthritis on Demodex mite infection. Arch Med Sci. 2018;14(2):353-356. doi:10.5114/ aoms.2016.60663

22. Biernat MM, Rusiecka-Ziolkowska J, Piatkowska E, Helemejko I, Biernat P, Gosciniak G. Occurrence of Demodex species in patients with blepharitis and in healthy individuals: a 10-year observational study. Jpn J Ophthalmol. 2018;62(6):628-633. doi:10.1007/s10384018-0624-3

\section{Publish your work in this journal}

Clinical Ophthalmology is an international, peer-reviewed journal covering all subspecialties within ophthalmology. Key topics include: Optometry; Visual science; Pharmacology and drug therapy in eye diseases; Basic Sciences; Primary and Secondary eye care; Patient Safety and Quality of Care Improvements. This journal is indexed on PubMed
Central and CAS, and is the official journal of The Society of Clinical Ophthalmology (SCO). The manuscript management system is completely online and includes a very quick and fair peer-review system, which is all easy to use. Visit http://www.dovepress.com/ testimonials.php to read real quotes from published authors. 